\title{
Le développement de cohérences transversales et verticales dans l'enseignement des langues étrangères
}

\section{Françoise Haramboure}

\section{(2) OpenEdition}

\section{Journals}

Édition électronique

URL : http://journals.openedition.org/asp/3172

DOI : 10.4000/asp.3172

ISSN : 2108-6354

Éditeur

Groupe d'étude et de recherche en anglais de spécialité

\section{Édition imprimée}

Date de publication : 1 décembre 1997

Pagination : 305-315

ISSN : 1246-8185

\section{Référence électronique}

Françoise Haramboure, «Le développement de cohérences transversales et verticales dans l'enseignement des langues étrangères », ASp [En ligne], 15-18 | 1997, mis en ligne le 13 novembre 2012, consulté le 01 mai 2019. URL : http://journals.openedition.org/asp/3172 ; DOI : 10.4000/ asp.3172

Ce document a été généré automatiquement le 1 mai 2019.

Tous droits réservés 


\title{
Le développement de cohérences transversales et verticales dans l'enseignement des langues étrangères
}

\author{
Françoise Haramboure
}

1 Engagée successivement dans l'enseignement de l'anglais dans l'enseignement secondaire et dans le secteur LANSAD ${ }^{1}$, aujourd'hui notre implication dans la formation des enseignants nous conduit naturellement à nous interroger sur les points de passage et de rupture entre ces deux moments de l'apprentissage des langues.

2 Se pencher sur cette problématique suppose que ces deux situations d'enseignement apprentissage des langues soient rigoureusement caractérisées. En terme chronologique, il s'agit de deux phases étroitement liées puisqu'elles s'inscrivent dans le prolongement l'une de l'autre. S'agissant du public, la majorité des élèves de l'enseignement secondaire deviennent des étudiants spécialistes d'autres disciplines. À ce titre, les compétences et performances des étudiants spécialistes d'autres disciplines peuvent être considérées comme révélatrices des acquisitions construites au cours de leur expérience d'apprentissage préalable. En matière de contextes, elles se situent dans des espaces d'apprentissage distincts, le collège, le lycée voir l'école élémentaire aujourd'hui pour la première, l'université et les classes post-baccalauréat pour la seconde, autant de lieux qui imposent à cet enseignement des contraintes spécifiques. En terme de durée, elles recouvrent des périodes de longueur différente correspondant à des temps différents du développement et de l'existence des individus concernés.

Dans ces conditions, ces deux étapes de l'apprentissage sont nécessairement marquées par des divergences sans pour autant exclure des convergences dès lors qu'on admet qu'elles s'inscrivent dans un continuum unique, celui du processus d'apprentissage des langues étrangères. 
4 Le but de cette réflexion est d'analyser les convergences et divergences entre ces deux phases de l'apprentissage afin de promouvoir des cohérences transversales et verticales susceptibles de le favoriser.

\section{Divergences et convergences}

\subsection{Les objectifs d'enseignement/apprentissage}

5 Indéniablement, les spécificités des contextes institutionnels dans lesquels se situent ces deux moments de l'enseignement apprentissage des langues ont une incidence sur les objectifs, les contenus et les démarches adoptées. Bien que les objectifs officiels de l'enseignement secondaire définis par l'institution, l'objectif communicationnel, l'objectif conceptuel, l'objectif culturel et depuis peu l'objectif méthodologique semblent à première vue identiques à ceux du secteur LANSAD, leur définition met en jeu des instances différentes. De ce fait, leur mise en oeuvre dans l'enseignement n'engage pas les différents acteurs au même degré.

\section{Une démarche «top down"}

6 Déterminés par la Direction des lycées et collèges, les objectifs sont sans aucun doute le reflet des connaissances actuelles en matière d'apprentissage des langues étrangères. Si les enseignants sont chargés de leur mise en oeuvre effective dans les classes, ils ne sont pas pour autant associés à leur élaboration. S'appuyant sur les résultats des recherches en matière de stratégies d'apprentissage et en matière de métacognition, l'introduction de l'objectif méthodologique en est une bonne illustration. Alors que l'objectif d'« apprendre à apprendre » était depuis un certain temps intégré en aval, il a fallu attendre les textes de 1995, concernant les programmes de la classe de sixième et plus largement «les langues vivantes au collège " pour qu'il soit explicitement reconnu. Pour autant, son opérationnalisation effective dans les classes suppose que les enseignants soient sensibilisés à la pertinence de cette question et reçoivent une formation préalable dans ce domaine, ce qui implique de nouveaux délais avant qu'il soit véritablement intégré dans les contenus et les démarches d'enseignement.

7 Autrement dit, il s'agit d'une approche « top down » qui néglige les acteurs responsables, les enseignants et les conditions de leur mise en oeuvre.

\section{Une démarche « bottom up »}

En revanche, les objectifs en aval ne dépendent pas d'instances extérieures au terrain, ils sont définis selon une démarche «bottom-up». Ils sont le produit d'une négociation entre les divers acteurs de la formation, les étudiants, les enseignants de langue et de communication, les responsables des formations concernées et, dans certains cas, les représentants de la profession. De ce fait, il s'agit d'objectifs locaux, en cohérence avec une formation donnée. À cet égard, l'expérience d'enseignement dans les DESS ou dans la formation des adultes montre que l'investissement des étudiants est directement proportionnel à leur implication dans la détermination des objectifs et à leur mise en oeuvre. Plus l'échéance de la vie professionnelle est proche plus la pression des étudiants est grande pour que les finalités de la formation en langue s'ajustent à leur projet individuel et global de formation.

Cette prise de responsabilité des étudiants ne signifie pas que la fonction de l'enseignant en langue soit plus limitée. Certes, son intervention se trouve modifiée dans la mesure où 
elle se fonde sur des initiatives partagées mais il n'en demeure pas moins qu'elle sollicite simultanément ses savoirs, savoir-faire et savoir-être. Ses savoirs et savoir-faire sont mobilisés dans l'interprétation des besoins/objectifs des étudiants en termes de besoins d'apprentissage et dans la didactisation des documents proposés. En effet, si l'étudiant est capable de déterminer ses besoins, il revient à l'enseignant de les traduire en contenus d'apprentissage. Ses savoirs disciplinaires interviennent également dans l'analyse des discours auxquels les étudiants seront confrontés et qu'ils seront appelés à produire. C'est de son savoir-être que dépendront l'existence et la réussite de ce partenariat entre enseignants et étudiants, la mise en oeuvre de collaborations transversales avec les enseignants des autres disciplines et l'accompagnement réussi de l'apprentissage.

\subsection{Le public}

\section{L'âge et le développement cognitif}

Sans doute, l'âge et par conséquent le développement cognitif sont en partie responsables des divergences entre ces deux phases de la formation en langue. Néanmoins, si nous nous référons aux stades du développement mis en évidence par Piaget, en leur donnant une interprétation souple tenant compte des variables individuelles, globalement, en termes de capacité d'apprentissage, les jeunes élèves se différencient davantage des élèves du second cycle que ces derniers des étudiants LANSAD.

11 En effet, les capacités d'apprentissage des jeunes élèves, notamment leurs capacités d'attention et d'abstraction sont différentes de celles des étudiants plus âgés. Pour les premiers, l'apprentissage exige le recours à l'action. Cette dernière favorise non seulement la compréhension mais aussi l'intériorisation et l'assimilation des formes grammaticales et lexicales en langue étrangère. L'accompagnement de l'expression et du geste correspondant en facilite l'acquisition. Parallèlement, leur sensibilité auditive leur permet de discriminer et de reproduire des sons, de percevoir des intonations et des rythmes qui échapperont à leurs aînés.

12 S'agissant de contenus, l'acquisition de connaissances lexicales, grammaticales et phonologiques est déterminante au début de l'apprentissage puisqu'elle conditionne la compréhension et l'expression en langue étrangère.

13 En revanche, les élèves dès la fin du 1er cycle, dans le second cycle et $a$ fortiori par la suite sont capables d'opérations formelles de plus en plus élaborées, c'est-à-dire d'élaborer des hypothèses, des inférences et des déductions. Les activités de compréhension, d'expression et de réflexion sur le fonctionnement de la langue, quel que soit le type de support choisi, y font constamment appel. Certes, en matière de compréhension et de production, les activités se complexifient peu à peu. S'il s'agit essentiellement pour les élèves du premier cycle d'interpréter des faits, le travail d'interprétation dépasse progressivement ce premier niveau pour dégager la portée implicite des informations présentées. Autrement dit, il n'y a pas de divergences fondamentales entre les capacités d'apprentissage des élèves du second cycle et celles de leurs aînés, le décalage se situe en amont.

\section{La motivation}

14 Le manque de motivation des élèves est sans doute le problème le plus fréquemment évoqué par les enseignants de langues dès la fin du premier cycle. L'observation de l'attitude des étudiants à leur arrivée à l'université ou dans les classes post-baccalauréat le confirme. À l'exception d'une minorité, c'est une stratégie d'évitement de l'échec qui 
caractérise leur stratégie d'apprentissage d'une langue étrangère. Dans cette logique, la préoccupation des élèves n'est pas tant de développer des compétences en langue étrangère de plus en plus élaborées pour améliorer leur performance que d'obtenir des résultats qui leur assureront le passage dans la classe supérieure ou la réussite au baccalauréat. Il ne s'agit donc pas d'une absence de motivation mais plutôt de « motivation extrinsèque » visant à éviter les répercussions d'une évaluation négative. Le déficit se situe au niveau de "la motivation intrinsèque ", liée à l'intérêt que l'on prend à faire une chose pour elle-même. Cette situation semble d'autant plus paradoxale si l'on considère l'enthousiasme que les jeunes débutants manifestent pour l'apprentissage de la langue étrangère.

Plusieurs explications peuvent être avancées. Sans doute l'organisation extensive de l'apprentissage sur sept ou huit ans, au terme desquels nombre d'élèves ont encore des difficultés à s'exprimer de façon autonome n'est pas étrangère à cet état de fait. L'évaluation directe de leur compétence en dehors du cadre scolaire ne les encourage pas à s'investir dans des activités d'apprentissage dont l'efficacité est peu probante. Les contraintes de l'enseignement collectif limitent les occasions de communiquer en langue étrangère en raison de la taille des groupes. Le caractère artificiel de la communication en classe de langue, renforcé le plus souvent par un schéma d'échange frontal, exclusivement contrôlé par l'enseignant aggrave les difficultés.

Pourtant ces raisons ne suffisent à expliquer ce déficit de motivation intrinsèque. Les outils conceptuels présentés par A. Lieury dans son dernier ouvrage (1996) permettent d'affiner l'analyse. Selon ses travaux, une forte motivation résulterait de deux besoins humains fondamentaux: la compétence perçue ou estime de soi et le sentiment d'autodétermination.

En matière d'estime de soi, la focalisation de l'enseignement des langues sur le code linguistique et par voie de conséquence sur les erreurs concernant notamment les savoirs déclaratifs d'ordre grammaticaux a deux effets négatifs sur la motivation des élèves : elle occulte leurs performances réussies et renforce l'image négative de leurs performances ; elle substitue à l'objectif communicatif partagé par la plupart des élèves un objectif strictement linguistique dont la pertinence est remise en question lorsqu'ils testent leurs compétences en dehors de l'école. Dans le contexte de la classe, l'enjeu n'est pas tant l'interaction en langue étrangère que l'acceptabilité linguistique. Il importe davantage pour l'élève de produire des phrases correctes en termes de syntaxe et de grammaire que de s'engager dans des échanges avec ses partenaires qui solliciteraient à la fois ses connaissances de la langue et ses stratégies.

18 S'agissant d'autodétermination, l'importance accordée aux activités de répétition, de reformulation ou de reproduction laisse peu de place à l'expression autonome de l'élève dans le premier cycle. Sans doute, ces activités, à la différence des exercices structuraux sont-elles contextualisées et contribuent-elles à faciliter la mémorisation des structures grammaticales et du lexique. Il n'en demeure pas moins qu'elles confèrent à l'élève plutôt un rôle de sujet enseigné que de sujet apprenant. Dans le second cycle, la prédominance du commentaire, qu'il s'agisse du commentaire de texte ou du commentaire de document iconographique, si stimulants que soient les supports choisis, ignore également la préoccupation communicative de l'élève au profit de l'utilisation de structures. Elle lui donne un statut de commentateur, contraint par les exigences de l'activité et le contenu du support, peu propice à la prise d'initiative et à l'expression en langue étrangère et peu susceptible de permettre à l'élève d'établir une relation autonome avec la langue qu'il 
apprend. Par ailleurs, le schéma des échanges organisés autour des questions de l'enseignant et des réponses des élèves limite leur dimension sociale. En ce sens, les conditions de production du discours court-circuitent la dimension interactive et sociale de la communication et de ce fait affectent la motivation des élèves à l'égard de l'apprentissage des langues étrangères.

Dans cette perspective, le concept de "washback", ou "backwash " nous parait particulièrement pertinent. Défini par M. Swain (1985) en ces termes « Washback refers to the effect of a test on teaching practices » il met en évidence les effets des modes d'évaluation sur les stratégies d'enseignement des enseignants et leur pratique. L'analyse des pratiques d'évaluation dans l'enseignement secondaire confirme notre interprétation. En raison de la difficulté à définir et à mettre en oeuvre des critères d'évaluation pour la compréhension et l'expression orale et de la place habituelle donnée à l'écrit dans l'évaluation, les enseignants d'anglais donnent la priorité à l'évaluation des performances écrites des élèves. Le poids des performances orales est marginal. Il en résulte qu'en amont, l'accent est également mis sur les activités écrites non seulement par les enseignants mais aussi par les élèves. Ces derniers adaptent leurs stratégies d'apprentissage au mode d'évaluation.

Cette orientation est accentuée par la nature des épreuves de langue au baccalauréat, c'est-à-dire au moment de l'évaluation terminale du second cycle. La nature écrite des trois épreuves obligatoires depuis 1995, compréhension écrite, compétence linguistique et expression écrite renforce la tendance évoquée tant en matière de stratégies et de contenus d'enseignement que de stratégies d'apprentissage, l'évaluation orale étant facultative. En termes de supports, dans le souci de mieux préparer les élèves à ce mode d'évaluation, les enseignants privilégient les textes écrits au détriment d'autres types de supports tels que les enregistrements audio et vidéo. En termes d'activités, la priorité est donnée aux activités de compréhension et de production écrites et aux stratégies correspondantes. Par voie de conséquence, les tâches de compréhension et de production orales sont négligées alors que les difficultés majeures des élèves en matière de compréhension se situent au niveau de la discrimination auditive, du repérage des formes faibles et des formes accentuées et de l'interprétation des phénomènes intonatifs. En ce sens, il existe une tension entre le projet fixé par l'institution et celui de la majorité des élèves, fût-il implicite, pour qui, sous la pression de la demande sociale en matière de langues étrangères, le développement de la compétence de communication et notamment sa composante orale est l'objectif prioritaire.

21 En raison de leur expérience d'apprentissage et devant les limites de leurs performances, les élèves manifestent une certaine résignation à l'égard de l'apprentissage de l'anglais à leur arrivée dans les classes post-bac. Cette attitude n'est pas sans incidence sur leur rapport à l'apprentissage de la langue étrangère. Celui-ci est marqué par une perception négative de leur aptitude à apprendre une langue étrangère et une certaine défiance à l'égard d'un système scolaire d'une efficacité peu probante dans ce domaine. Dès lors, le problème qui se pose aux enseignants du secteur LANSAD est celui de créer une nouvelle motivation chez les étudiants concernés, comme nous l'avons souligné précédemment.

Les propositions élaborées par divers enseignants chercheurs travaillant dans le domaine LANSAD sont convergentes (Breton 1996; Haramboure 1996; Sabiron 1996), quelle que soit l'organisation de l'enseignement choisi. Qu'il s'agisse d'un apprentissage présentiel, en autonomie semi-guidée ou guidée, les réponses avancées ont une caractéristique commune: l'articulation autour d'un projet fondé sur la réalisation de tâches 
communicatives interdépendantes, micro-projets ou scénarios visant à résoudre le problème déjà évoqué de la pratique de la langue étrangère.

L'analyse de ces dispositifs, malgré leur diversité apparente, fait apparaître des caractéristiques communes. Ils répondent à la demande explicite ou implicite des étudiants d'apprendre à communiquer dans la langue étrangère puisque chacune des tâches proposées vise une des composantes de la capacité de communication, compréhension écrite et orale, production écrite et orale et des activités qui y participent telles que la conceptualisation et la métacognition. Ils placent l'étudiant en situation d'acteur puisque la réussite de chaque tâche engage la mise en oeuvre de stratégies spécifiques et transférables sans exclure le recours à des stratégies personnelles. Par exemple si la stratégie d'anticipation nécessaire à la compréhension reposera chez certains sur la prise rigoureuse d'indices et leur association avec leur connaissance du thème abordé, elle pourra faire appel chez d'autres à l'intuition et à l'imagination.

Dans la logique d'une pédagogie de projets, il s'agit de dispositifs intégrés et souples dont la finalité est commune. Les informations linguistiques ou extra-linguistiques recueillies au moment de la compréhension sont réinvesties au moment de la production, les faits de langue rencontrés ou produits sont le point de départ des activités de conceptualisation et les activités métacognitives amènent l'élève à réfléchir sur les stratégies qu'il a adoptées au cours des activités précédentes.

L'interaction orale y retrouve sa place sous des formes variées, simulations d'entretiens ou de négociations, jeux de rôles, discussions de sondages, brainstorming, etc., autant d'activités qui permettent de diversifier le schéma habituel de la circulation de la parole en cours de langue. Elle a pour effet de réactiver et de développer conjointement les savoirs lexicaux, grammaticaux, phonologiques et culturels et les stratégies des étudiants.

Dans certains contextes, notamment dans le cadre de formations courtes, par alternance ou centrées sur la gestion de projet, le projet de formation en langue s'intègre étroitement au processus de formation dans la spécialité. Dans ce cas, le plus souvent d'ailleurs à la demande des étudiants, les supports de compréhension recouvrent les thématiques des autres disciplines. Ils sont sélectionnés par les étudiants avec le concours des enseignants spécialistes et des enseignants de langue. Les activités de compréhension deviennent une recherche finalisée d'informations, orientée vers la résolution d'un problème extra-linguistique, à savoir l'approfondissement de leurs connaissances dans une de leurs spécialités ou la recherche d'informations pour la réalisation d'un projet extra-linguistique par exemple.

Le format des interactions s'inscrit dans la même dynamique: exploitant les connaissances lexicales, grammaticales auxquelles les étudiants ont été confrontés au cours des activités de compréhension, elles sont conçues de façon à les amener à mettre en oeuvre les stratégies susceptibles de résoudre les problèmes d'expression telles que la rédaction d'un message électronique, d'un abstract, la présentation orale d'un rapport d'étape ou d'un projet. L'intérêt de ce dispositif est qu'en raison de sa dimension interdisciplinaire, il répond à des besoins immédiats et explicites des étudiants tout en leur donnant les outils méthodologiques pour satisfaire leurs besoins en langue étrangère à long terme. Il est à l'origine d'un regain de leur motivation puisqu'il valorise leur sentiment de compétence et leur sentiment d'autodétermination quant à l'orientation de la formation en langue. 

mettre en relation l'apprentissage de la langue étrangère avec ses pratiques sociales, à savoir la communication en langue étrangère. Ce faisant, elle lui donne d'autant plus de sens qu'elle répond aujourd'hui à une demande sociale forte amplifiée par la construction européenne et le contexte socio-économique, demande que reflètent les objectifs des élèves en matière d'apprentissage des langues. Elle s'appuie sur une dynamique visant à recréer dans le cadre de la classe, à travers l'élaboration de tâches adaptées, les conditions authentiques de mise en oeuvre des savoirs et savoir-faire spécifiques à la communication en langue étrangère. Elle concourt à lever l'obstacle évoqué par $\mathrm{Ph}$. Meirieu (1989) lorsqu'il affirme qu' :

un savoir qui n'est pas articulé à une pratique et qui ne se présente pas comme pouvant résoudre les problèmes qu'elle soulève ne peut pas faire l'objet d'une appropriation. L'intégration des tâches est sans doute la clef de cette démarche puisqu'elle est le gage de
leur orientation vers un but univoque, le développement de la compétence en langue étrangère. Pour autant, elle n'implique pas la rigidité. Au contraire, il importe que les varier.

De ce fait, l'interaction n'y est plus marginalisée. Elle retrouve sous la forme d'activités variées présentées précédemment le rôle qu'elle occupe naturellement dans la pratique de la langue. Elle satisfait le désir souvent observé chez les élèves de communiquer entre eux sur des thèmes qui relèvent de leur expérience et qui font sens pour eux. Ses schémas habituels sont remis en cause puisqu'elle repose moins sur des échanges convenus entre l'enseignant et l'élève que sur une communication entre élèves, sollicitant leur stratégie

$$
\begin{aligned}
& \text { écr } \\
& \text { élève } \\
& \text { les } \\
& \text { int } \\
& \text { d'u } \\
& \text { var } \\
& \text { De } \\
& \text { var } \\
& \text { de } \\
& \text { ha } \\
& \text { l'e }
\end{aligned}
$$


de négociation, de coopération et de compensation pour résoudre un problème, l'enseignant s'effaçant pour intervenir à la demande des élèves. La longueur et la complexité des activités varient en fonction du niveau des groupes.

Dans cette optique, l'interaction remplit une fonction double: elle contribue au réinvestissement des savoirs, des savoir-faire et des savoir-être des élèves à condition que l'enjeu de l'activité soit suffisamment stimulant et elle facilite la confrontation de leurs perceptions de faits linguistiques ou extra-linguistiques, confrontation qui peut être à l'origine d'ajustements et de constructions de nouveaux savoirs et savoir-faire, selon les travaux d'A. N. Perret-Clermont.

\subsection{Les contenus}

En termes de contenus, la dimension interdisciplinaire et la composante interculturelle semblent porteuses de nouvelles cohérences entre l'enseignement secondaire et le secteur LANSAD en tenant compte de leur contexte propre.

\section{La dimension interdisciplinaire}

En mettant en relation le fonctionnement de la langue maternelle et celui des langues étrangères étudiées, selon les principes de l'éveil au langage élaboré par Hawkins(1987) les élèves découvriront que chacune des langues étudiées constitue un système indépendant faisant appel à un système phonologique, un lexique, des règles de fonctionnement distincts. Cette mise en relation leur permettra de se départir de leur attitude ethnocentrique qui les conduit à considérer le fonctionnement de leur langue maternelle comme universel et par conséquent à le transposer dans le fonctionnement de la langue étrangère. Ils prendront également conscience qu'une langue n'est pas un catalogue de formes correspondant terme à terme avec celles d'autres langues et que par conséquent le transfert systématique des formes de la langue maternelle peut conduire à des erreurs. Dans cette optique, l'utilisation d'une métalangue unifiée pour analyser le fonctionnement des diverses langues contribuera à en faire saisir aux élèves les caractéristiques spécifiques.

Par ailleurs, la pratique de la compréhension et de l'expression dans les diverses langues leur permettra de saisir que ces opérations mobilisent des stratégies transversales communes quelle que soit la langue envisagée et en facilitera leur procéduralisation. Elle leur permettra aussi d'envisager la dimension extra-verbale de la communication, par exemple, la portée du geste lorsque surgit un problème et sa dimension culturelle.

Dans cette dynamique, le croisement des connaissances acquises dans les autres disciplines, telles que l'histoire, la géographie et les disciplines artistiques par exemple, avec les savoirs que les élèves construisent par l'apprentissage de la langue étrangère, enrichira le capital culturel dont ils disposent sur la culture étrangère.

\section{La composante interculturelle}

Le contact avec les diverses langues et la mise en relation de leurs spécificités doit entraîner l'émergence d'un troisième type de cohérence dans l'apprentissage des langues étrangères, à savoir la composante interculturelle, concept dont la portée complémentaire a été soulignée par M. Byram (1992) lorsqu'il affirme qu'il existe

trois fils conducteurs interdépendants dans l'enseignement d'une langue étrangère : la pratique de la langue, la conscience de la nature de la langue et la compréhension des cultures étrangères et maternelles 

langue soit envisagée comme une des expressions de la culture étrangère. À ce titre, l'apprentissage $d u$ lexique et l'étude $d u$ fonctionnement des langues étrangères permettent la mise en évidence d'un découpage différent de la réalité. D'autre part, que la mise en contact avec la culture cible soit exploitée pour développer chez les élèves une prise de conscience de leur culture maternelle. Pour ne citer qu'un exemple de l'incidence de l'approche interculturelle sur la communication en langue étrangère, analysons ses effets sur la compréhension. Processus interactif entre le support en langue étrangère et le lecteur/auditeur, elle suppose l'exploitation par ce dernier d'indices contextuels situés culturellement et l'utilisation de ses connaissances et expériences marquées par sa culture maternelle. En ce sens, l'interprétation se fonde sur une décentration à l'égard de sa propre culture faute de quoi les événements présentés seront envisagés à travers le filtre de sa culture maternelle. Autrement dit, elle engage conjointement la culture cible et la culture source et se fonde sur un regard interculturel.

Cette dimension aura pour effet de ne pas dissocier l'enseignement de la langue celui de la culture étrangère. De ce fait, la connaissance de la culture étrangère ne reposera plus seulement sur l'acquisition de savoirs distincts et disparates venant se superposer aux savoirs sur la langue, elle fera partie intégrante de l'étude de la langue.

\section{Conclusion}

Bien qu'il s'agisse d'un processus unique dont les objectifs sont identiques, nombreux sont les clivages entre l'enseignement/apprentissage des langues dans le secondaire et le secteur LANSAD. Sans doute certains relèvent-ils du développement intellectuel des élèves. Néanmoins, les solutions proposées pour l'apprentissage des langues par les étudiants spécialistes d'autres disciplines semblent répondre aux préoccupations de ces deux types de public. En ce sens, la démarche de projet, l'intégration explicite d'approches interdisciplinaires et interculturelles contribuent au développement de cohérences transversales et verticales susceptibles d'apporter des solutions aux problèmes qui se posent aujourd'hui dans l'enseignement secondaire et d'enrichir les démarches adoptées dans le secteur LANSAD.

\section{BIBLIOGRAPHIE}

Breton, L. « Rôle des représentations des apprenants ». ASp 11/14, 1996, pp. 307-312.

Byram, M. 1992. Culture et éducation en langue étrangère. Paris : Didier LAL.

Haramboure, F. 1996. « La démarche de projet dans l'enseignement /apprentissage d'une langue étrangère ». ASp 7-10, 193-205.

Isani, S. 1996. « Le scénario comme outil pédagogique ». ASp 11-14, 313-326.

Hawkins, E. 1987. Awareness of Language: An introduction. Cambridge : Cambridge University Press. 
Lieury, A. 1996. Motivation et réussite scolaire. Paris : Dunod.

Meirieu, Ph. 1989. Enseigner, scénario pour un métier nouveau,. Paris : ESF éditeur.

Perret-Clermont, A.N. 2000. La construction de l'intelligence dans l'interaction sociale. $5^{\mathrm{e}}$ éd. Berne :

Peter Lang.

Sabiron, J. 1996. « Cohérences plurielles ». ASp 11-14, 1996, 239-252.

Swain, M. 1985. « New directions in language testing ». In Large-scale Communicative Language

Testing: A case study. Oxford : Pergamon.

\section{NOTES}

1. LANSAD : langues pour spécialistes d'autres disciplines

\section{RÉSUMÉS}

Si l'apprentissage d'une langue étrangère relève d'un processus continu, les formations en anglais général dans l'enseignement secondaire et en anglais de spécialité à l'université contribuent à un processus identique. Tenant compte des caractéristiques de chaque contexte d'apprentissage, cet article examine les convergences et les divergences entre ces deux niveaux, analyse leur influence sur la motivation des étudiants, les stratégies d'enseignement et d'apprentissage et enfin propose une démarche plus cohérente.

If foreign language learning forms a continuous process, then EGP courses at secondary school level and ESP courses at university level contribute to the same process. Taking into account the characteristics of each learning context, this paper explores the similarities and differences between these two levels, assesses their impact in terms of students' motivation and teaching and learning strategies and suggests a more coherent approach.

\section{INDEX}

Mots-clés : anglais de spécialité, anglais général, cohérence, enseignement secondaire, enseignement universitaire

Keywords : coherence, EGP, English for Specific Purposes, ESP, secondary school level, university level

\section{AUTEUR}

\section{FRANÇOISE HARAMBOURE}

Françoise Haramboure est maître de conférences à l'UFR des pays anglophones, Université Michel-de-Montaigne Bordeaux 3, où elle enseigne la didactique des langues étrangères après avoir exercé dans le secteur LANSAD. Elle est responsable de la préparation à l'épreuve sur 
dossier pour le CAPES d'anglais dans le cadre de l'IUFM d'Aquitaine. Elle intervient dans le DEA de langue anglaise des spécialités scientifiques et techniques. Elle assure l'enseignement de l'anglais dans une formation DESS de Gestion de projet. f.haramboure@cegetel.net 